\title{
Goldenhar Syndirome
}

Priti SL, Mulimani MS, Nivedita K

Department of General Medicine, Shri B.M. Patil Medical College Hospital and Research Centre, Vijayapura, India.

Abstract:

A 20 year old male patient presented with ascites since birth, swelling of right scrotum, absence of development of secondary sexual characters, breathlessness on exertion, short limb gait, and hematemesis. Ultrasound imaging and CT angiography of the abdomen revealed absence of portal vein, thrombus at the junction of superior mesenteric vein and splenic vein, cavernous transformation and development of collaterals. Echocardiography revealed hypertrophic obstructive cardiomyopathy (HOCM) and X-ray hip revealed absence of head of femur. Scrotal examination revealed right sided indirect inguinal hernia and hydrocoele. A diagnosis of Goldenhar syndrome was made.

Goldenhar syndrome consists plethora of clinical features comprising congenital portal vein absence with associated cardiac defects, skeletal abnormality and urogenital defects. Our case deserves attention and needs to be reported because so far in the medical literature only one case of congenital portal vein absence with HOCM has been reported and also no other case of congenital portal vein absence with thrombosis at the SMV and SV junction associated with HOCM has been reported in the medical literature.

Key words: Goldenhar Syndrome, Hematemesis, Hypertrophic Cardiomyopathy, Inguinal Hernia, Scrotum.

\section{Introduction}

Congenital abnormality of portal vein associated with cardiac, genitourinary and musculoskeletal abnormality is a rare entity $[1,2]$. Congenital portal vein anomaly was first reported by Abernathy in 1793 [3]. Goldenhar reported congenital portal vein abnormalities, including cardiac, musculoskeletal and genitourinary abnormality and named it Goldenhar syndrome. Less than 200 cases have been reported in the medical literature so far and only one case of HOCM associated with portal vein anomaly has been reported previously by RD Bellah and colleagues [2]. Our case report is of clinical significance deserves attention and needs to be reported in the medical literature.

\section{Case Report}

A 20 year old male presented with distention of abdomen since birth, short limb gait, absence of development of secondary sexual characters,

\section{Corresponding Author: Dr. Priti SL}

Email: pritisI2011@gmail.com

Received: June 23, 2015 | Accepted: August 4, 2015 | Published Online: August 30, 2015

This is an Open Access article distributed under the terms of the Creative Commons Attribution License (creativecommons.org/licenses/by/3.0)

Conflict of interest: None declared | Source of funding: Nil | DOl: http://dx.doi.org/10.17659/01.2015.0095 
scrotal swelling, breathlessness on exertion since 2 months and two episodes of hematemesis 6 months ago. His past medical history involves consultation with various doctors for the complaints of abdominal distension without relief of symptoms and also had a past history of admission to hospital due hematemesis and undergone variceal ligation. No history of similar complaints in the family was noted.

General physical examination revealed blood pressure of $110 / 70 \mathrm{~mm} \mathrm{Hg}$, pulse: $80 / \mathrm{min}$, no pallor or icterus. Absence of facial, axillary, chest and pubic hair, gynaecomastia was present. Bilateral parotid enlargement was present. Right scrotal swelling on examination revealed indirect inguinal hernia and congenital hyrocoele. Moderate ascites, palpable spleen, engorged veins over the abdomen with direction of flow from below upwards was seen. Cardiovascular system examination showed palpable apex impulse at $6^{\text {th }}$ intercostal space $1 / 2$ inch lateral to the midclavicular line, character of apex impulse was heaving type, S1 and S2 were normal, high pitched pansystolic murmur of grade 3 intensity in the mitral area and ejection systolic murmur of grade 3 intensity in the aortic area were present. Respiratory and CNS examination were normal.

Investigations revealed hemoglobin: 9.1 gm\%, PCV: $28.5 \%$, MCV: 66.3 fl, platelet count: 0.84 lakhs $/ \mathrm{mm}^{3}$, ESR: $15 \mathrm{~mm}$ at $1^{\text {st }}$ hour, serum creatinine: $0.8 \mathrm{mg} / \mathrm{dL}, \mathrm{Na}^{+}: 132 \mathrm{meq} / \mathrm{L}, \mathrm{K}^{+}: 4.2$ $\mathrm{meq} / \mathrm{L}$. Liver function test revealed low serum albumin of 2.8, rest of LFT was normal. Hepatitis $C$ antibodies and hepatitis $B$ antigen were nonreactive. ECG showed left ventricular and atrial enlargement. An endoscopy revealed grade II esophageal varices.

Ultrasound abdomen showed nonvisualization of portal vein with multiple collaterals at the porta hepatis suggestive of portal vein thrombosis with portal cavernoma formation,

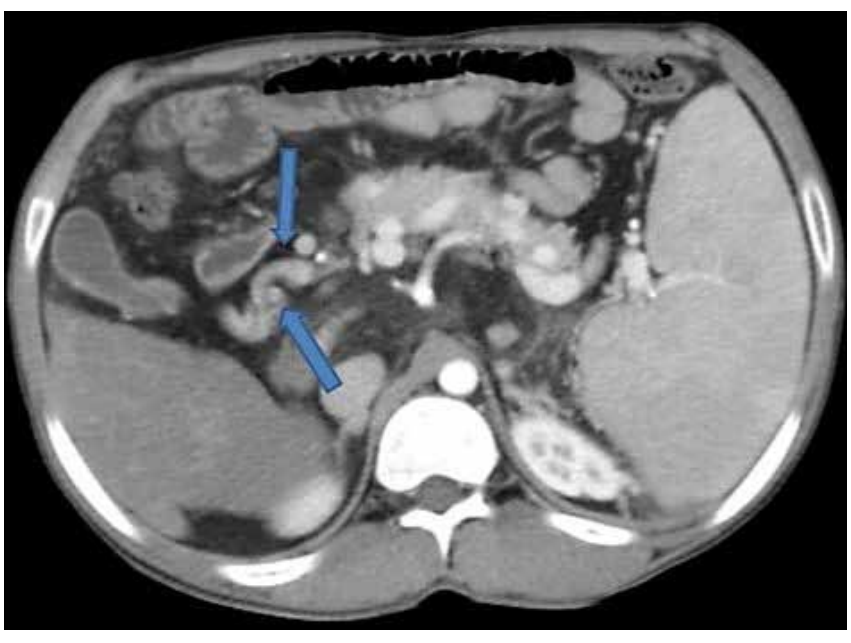

Fig.1: CT contrast showing multiple collaterals at porta hepatis with non-visualization of porta hepatis.
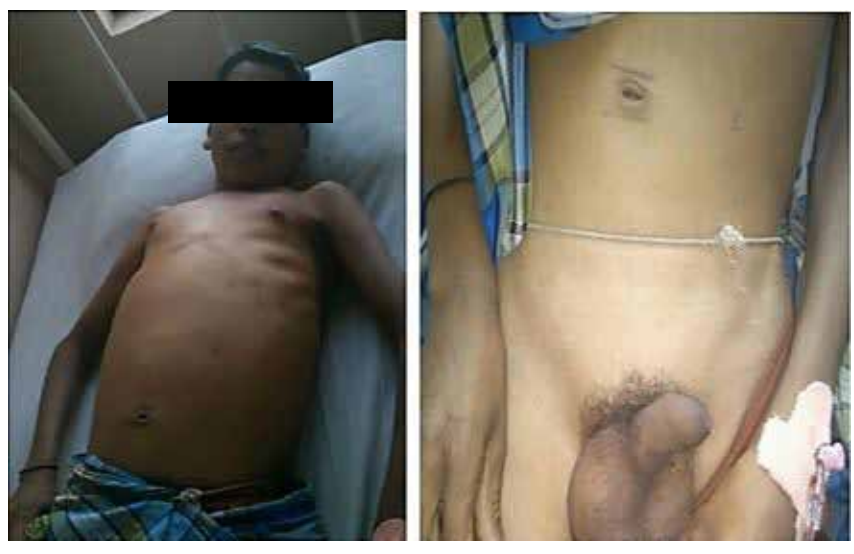

Fig.2:(a) Figure showing ascites and gynaecomastia (b) Right sided inguinal hernia with hydrocele.

periportal and gall bladder fibrosis, splenomegaly with multiple collaterals at the splenic hilum and moderate ascites. CT abdomen with contrast revealed similar findings, additionally it showed the absence of left head of femur.

$\mathrm{ECHO}$ revealed asymmetrical septal hypertrophy, grade II eccentric mitral regurgitation, dilated left atrium, left ventricular outflow tract 
obstruction with gradient of $51 \mathrm{~mm} \mathrm{Hg}$ and good ventricular function suggestive of hypertrophic obstructive cardiomyopathy. The patient was managed conservatively with propranolol $40 \mathrm{mg}$, hematinics and on discharge he was advised to report to the hospital immediately if he had any hematemesis episodes.

\section{Discussion}

Congenital abnormalities of the portal venous system are rare. Few previous reports have described abnormal portal embryogenesis as the cause for portal vein anomaly with cardiac, skeletal and genitourinary defects. Knowledge of the embryological development of portal vein is necessary to understand the associated anomalies. The portal vein develops between $4^{\text {th }}$ and $10^{\text {th }}$ weeks. In the four week embryo, three paired venous systems are present, these are umbilical veins of chorionic origin, vitelline veins from the yolk sac and cardinal veins from the body of the embryo. Towards the end of the $4^{\text {th }}$ week three cross links are formed between the right and left vitelline veins. The intrahepatic portal veins develop from the superior link, while the extrahepatic portal vein is formed by the selective involution of the caudal part of the right vitelline vein and cranial part of the left vitelline vein. Primary failure to form this critical anastomosis may lead to complete or partial absence of the portal system [4].

Congenital anomaly of portal vein is one of the causes of portal vein thrombosis. Congenital abnormality of the portal vein was $1^{\text {st }}$ described by Abernathy in 1793 [3]. Subsequent to this report further less than 200 cases have been reported. Several associated congenital abnormalities were also described which were associated with congenital portal vein anomaly i.e. cardiac defects like ASD, VSD, PDA, aortic stenosis, pulmonary stenosis, inferior vena cava and superior venacaval obstruction, skeletal abnormalities like dislocated hip, arachnodactyly and urogenital abnormalities like cystic dysplasia of kidneys, vesico-urinary reflux, hypospadias and inguinal hernia [3]. $M$. Odivre and colleagues conducted a study of 30 children with extrahepatic portal hypertension and found that $40 \%$ of the patients with congenital portal vein anomaly had these associated cardiac, musculoskeletal and genitourinary abnormalities [5].

\section{In 1989 R.D Bellah and colleagues} reported a case of portal vein abnormality with fatal congenital hypertrophic cardiomyopathy [2]. The close relationship between the development of vitelline veins and the heart in embryonic life may be responsible for the association between the cardiovascular malformations and congenital abnormality of portal vein [6].

\section{Conclusion}

An awareness of the possibility of associated congenital abnormalities should also be kept in mind when we come across any patient with extrahepatic portal vein hypertension. Such patients must be treated like any other extrahepatic portal vein obstruction patients with propranolol, management of variceal bleeding by endoscopic variceal ligation, thrombosis need not be treated as it is chronic and already collaterals were developed [7]. Major abnormalities in multiple system might contraindicate any major surgical procedure in such patients.

\section{References}

1. De Gaetano AM, Gui B, Macis G, Manfredi R, Di Stasi C. Congenital absence of portal vein associated with focal nodular hyperplasia in the liver in adult women: imaging and review of the literature. Abdomen imaging. 2004;29:455459.

2. Bellah RD, Hayek J, Littlewood R. Anomalous portal venus connection to supra hepatic vena 
cava: sonographic demonstration Paediatr Radiol. 1989;20:1 15-117.

3. Abenathy J. Account of two instances of uncommon formation in the viscera of the human body. Philos Trans R Soc. 1793;83:59-66.

4. Howard ER, Davenport M. Congenital extrahepatic portocaval shunts-the Abernethy malformation. Paediatr Surgery. 1997;32;494497.

5. Odilvre $M$, Pigs $G$, Alagille D. Congenital abnormalities associated with extrahepatic portal hypertension. Archives of Disease in Childhood. 1977;52:383-385.

6. Gocman R, Akhan O, Talim B. Congenital absence of the portal vein associated with congenital hepatic fibrosis. 2007:37;920-924.

7. Arora NK, Das MK. Extra hepatic portal venous obstruction in children. Available at: http:// cdn.intechopen.com/pdfs-wm/32248.pdf Accessed on 23 June 2015. 